\title{
Interactive comment on "Facility scale inventory of dairy methane emissions in California: Implications for mitigation" by Alison R. Marklein et al.
}

\section{Anonymous Referee \#1}

Received and published: 9 October 2020

The paper reports the first (to my knowledge and as stated by the authors) spatiallyexplicit database of dairies in California. Such detailed information is of great relevance for improving bottom-up emissions estimates, comparing bottom-up to top-down methods and assessing the effectiveness of mitigation options. The method is written up in a transparent way that will also allow relatively easy adjustment of individual parameters in the future.

The paper is generally clearly written, but also relatively long. I sometimes got a bit lost in paragraphs where the individual parameters are described one after the other and suggest that you refer to the tables more efficiently, if possible. The underlying 
choices are substantiated by relevant references to existing literature or databases. There are some places where information from the introduction (motivation) is repeated in results, and in particular in the conclusions section. I encourage the authors to reduce repetitions and focus in the conclusions mainly on the results from this study, or otherwise shorten these paragraphs in the introduction.

My second request is to pay attention to the units, they do not always fit throughout the paper (some omissions in the text, which seem to be correct in the tables).

Specific suggestions:

Line 150-155: Is it really necessary to list all the counties? I suggest to delete this and refer to Table S2.

Line 232: unit: should be $\mathrm{kg} / \mathrm{cow} / \mathrm{yr}$, correct in Table, all numbers in these paragraph miss the time interval in the denominator (per year)

Line 243 and at other places: It is mathematically correct to add the letter $i$ under the sum symbol to indicate the parameter that is summed over

Line 248: units: DMI should be $\mathrm{kg} / \mathrm{cow} /$ day, value and unit $(\mathrm{g} / \mathrm{kgDMI})$ should be stated in the text for E2

Line 257: "and" missing before milkfat

Line 257: ... we also include emission factors

Line 260: The index "l" is missing for mf and f_mf

Line 267: To make the units fit in the equation, you should multiply by 365 days/yr

Line 282: Density: unit is wrong, must be $\mathrm{g} / \mathrm{l}$, or $\mathrm{g} / \mathrm{dm} 3$

Printer-friendly version

Line 283: Provide value and unit for $\mathrm{B} \_0$, describe what the Max $\mathrm{CH} 4$ production capacity is

Line 284: Refer to Tables here. Are the VS values in tables?

Interactive

comment 
Line $296 \mathrm{ff}:$ Refer to tables for better overview

Linne 299: B_0: 0 should be subscript

ESSDD

Line $299 \mathrm{ff}$ : for MCF refer to tables for better overview

Page 13; line numbers missing in my copy, line 1: Are these units correct? More than 2 tons per day? Add B_0 values to a table.

Interactive

comment

Line 341: I think it is adequate to neglect the variation of methane density, but is this also true for maximum methane production? Please also clarify what this parameter is, see comment line 283.

Line 359, stick to symbol rho for density as in other equations

Line 404: seems to be a typo, value and error are exactly the same number

Line 423: I suggest that you may want to comment on whether or not the smaller uncertainty of M1 means that method M1 is better than methods M2 and M3, and what could be the reason for this large difference in uncertainty.

Line $439 \mathrm{f}$ : the value that is described as highest here, 0.25 for Central Valley, is smaller than the 1.6 for the North Coast. Must be a mix up of numbers.

Line 448: Full stop missing

Line 449: ... differences in enteric : manure emissions ...

Line 525: leave out this sentence? You implicate in the next sentence that the statewide numbers can also be derived with the older methods (maybe add somewhere tin the discussion earlier that this is not so surprising at least for enteric, since you use the same numbers of animals).

Line 531-536: Repeated from introduction (motivation) and not necessary to repeat here.

Line 539-542: Repeated from method/results and not necessary to repeat here. 
Line 545-554: shorten/reword this paragraph focused to the sentence; We are most confident... you could strengthen the point that better reporting would help for your ESSDD new inventory. Sentence with reference to Hamilton could be moved to methods.

Table 3: Make sure that units reported are consistent (factor 1/cow)

Table 4: Provide units for all parameters

Interactive

comment

Line 598: delete "the ratio of"

Figure 4: describe unit of color bar $(-0.3$ to 0.3$)$. Are these relative or absolute differences?

Line 615: M3 should be Calgem

Supplementary material:

L24: water board reports

L33: ... counties that did not

L34 ff: do you need this long list of counties (refer to table?

Line $64 \mathrm{ff}$ : Maybe you can make the point that $\mathrm{n}$ is actually the easiest variable to improve knowledge on, so better constraints on $n$ will be valuable especially since some final numbers depend strongly on $\mathrm{n}$. This also holds for dairies in VISTA.

Line 84: Not clear why SE is based on $\mathrm{n}=77$

Line 104: Can you illustrate for this one concrete example explicitly how you come from the two values $69.8 \%$ and $78.2 \%$ to the standard error of $26.2 \%$ ? This is not immediately clear to me.

Line 106, 114, and few other places: correct mathematical way of writing this result is $(34.0+/-12.0) \%$

Line 131: but also for E2, M1, and M2 in S1.2.2: Provide the final value for SE in text, 
or refer to table. Now you only state the confidence bounds, but not the final SE.

Line $160 \mathrm{ff}: \mathrm{S} 2.1$ could potentially be a table in the main paper, would allow to shorten ESSDD text there

Line $169 \mathrm{ff}:$ S2.2 could be shown along Fig. 1 in the main paper

Line $183 \mathrm{ff}$ : I strongly suggest that S2.3 should be shown in the main paper, units

Interactive

comment should be added, and for constant values also the values here.

Line 185: you use rho rather than density in the equations, adapt here

Interactive comment on Earth Syst. Sci. Data Discuss., https://doi.org/10.5194/essd-2020-133, 2020. 